\title{
Molecular dynamics computer simulation of amorphous silica under high pressure
}

\author{
J Horbach \\ Institut für Materialphysik im Weltraum, Deutsches Zentrum für Luft- und \\ Raumfahrt (DLR), 51170 Köln, Germany \\ E-mail: juergen.horbach@dlr.de
}

\begin{abstract}
The structural and dynamic properties of silica melts under high pressure are studied using molecular dynamics (MD) computer simulation. The interactions between the ions are modeled by a pairwise-additive potential, the so-called CHIK potential, that has been recently proposed by Carré et al. The experimental equation of state is well-reproduced by the CHIK model. With increasing pressure (density), the structure changes from a tetrahedral network to a network containing a high number of five- and six-fold Si-O coordination. In the partial static structure factors, this change of the structure with increasing density is reflected by a shift of the first sharp diffraction peak towards higher wavenumbers $q$, eventually merging with the main peak at densities around $4.2 \mathrm{~g} / \mathrm{cm}^{3}$. The self-diffusion constants as a function of pressure show the experimentally-known maximum, occurring around a pressure of about $20 \mathrm{GPa}$.

PACS numbers: 61.20.Ja,64.70.ph,66.30.H-,62.50.-p
\end{abstract}

\section{Introduction}

Under ambient conditions, amorphous silica $\left(\mathrm{SiO}_{2}\right)$ forms a disordered tetrahedral network. Tetrahedral units with silicon atoms in the centre are connected to each other via the oxygen atoms at the four corners of each tetrahedron. Under compression, the network structure of $\mathrm{SiO}_{2}$ may change significantly. From experiments on silica glasses 1, 2, 3, 4, 5, 6, 7, it is known that the $\mathrm{SiO}$ coordination changes for pressures of the order of 15-20 GPa. Then, the network structure is dominated by five- and six-fold coordinated $\mathrm{SiO}$ units. Albeit of great interest for geoscience, experimental studies of pure silica melts under (high) pressure are very rare [6]. This is due to the high temperatures that are required to study silica in its liquid state. Hence, computer simulations are an important tool to elucidate structural and dynamic properties of silica melts at high pressure.

In many of the recent molecular dynamics (MD) computer simulations, the pair potential proposed by van Beest, Kramer and van Santen, the so-called BKS potential [8, has been used to model the interactions between the $\mathrm{Si}$ and $\mathrm{O}$ ions. Tse et al [9] showed that in BKS silica the oxygen coordination around silicon atoms changes from 4 to 5 at intermediate pressures $(10-15 \mathrm{GPa})$ and reaches 6 at high pressures. Subsequent studies of BKS silica [10, 11, 12] have indicated that transport processes first become faster with increasing pressure, in agreement with experimental studies on silicate melts [13, 14, 15, 16]. In Ref. [10, this was interpreted as a signature of a 
strong-to-fragile transition, according to Angell's classification of glassforming liquids 17, 18. Note that the anomalous transport in silica under pressure was already seen in a pioneering MD simulation by Angell et al [19] using a different model potential. Further studies on BKS silica [20, 21, 22, 23] addressed the question to what extent the properties of silica are typical for a strong glassformer, sharing its properties with other network-forming liquids such as water. In Ref. [20], the possibility of a phase separation between a low and high density liquid phase was discussed and a rough estimate of the free energy as a function of volume and temperature was used to locate such a transition for BKS silica, finding a critical point at a density of about $2.9 \mathrm{~g} / \mathrm{cm}^{3}$ and a temperature of about $2000 \mathrm{~K}$.

But recent works have called in question that simulations with simple pair potentials can give reliable results for pressure-induced phenomena in silica. Trave et al 25. claimed that empirical force fields such as the BKS model "are not sufficiently accurate to reproduce pressure-induced phenomena such as changes in coordination and increasingly strained geometries". These authors used "first-principles" CarParrinello molecular dynamics (CPMD) simulations to investigate liquid silica for pressures up to about $30 \mathrm{GPa}$ at a constant temperature of $T=3500 \mathrm{~K}$. In a CPMD, the electronic degrees of freedom are taken into account in the framework of a density functional theory [24] and thus it is not relying on the use of effective interatomic potentials. Whereas the CPMD calculation for silica shows good agreement with respect to the experimental equation of state, the BKS model underestimates the experimental specific volume systematically by about 15\%. Based on CPMD calculations, Tangney and Scandolo (TS) [26] have developed a new effective potential that nicely reproduces the experimental equation of state of amorphous silica as well as some of the properties of crystalline phases [27. The TS model is a fluctuating dipole moment potential, accounting for the polarizability of the oxygen atoms. A disadvantage of the TS model is its low efficiency. Due to the expensive self-consistent computations of the dipole moments on the oxygen atoms in every time step, up to two orders of magnitude more computer time is needed in MD simulations with the TS model than for comparable simulations with a simple pair potential such as the BKS model [27].

The conjecture of Trave et al about the validity of simple effective interaction models for silica has been recently challenged by Carré et al [28] who developed a new pair potential for silica, the so-called CHIK potential. The CHIK potential was also parametrized from CPMD calculations and was shown to be superior to the BKS model with respect to various properties of amorphous silica (in particular the density at low pressures) [28. In this work, we show that it also reproduces very accurately the experimental equation of state, various structural properties and the anomalous diffusion dynamics of silica under pressure. This proves the transferability of the CHIK potential to the description of amorphous silica under high pressure. Thus, the CHIK model can be used to readdress the questions about the possibility of a liquidliquid phase separation and the mechanisms of anomalous transport phenomena at high pressures.

\section{The model potential and details of the simulation}

At first glance, it might be surprising that the directional covalent bonding in silica can be described by an interaction model that depends only on the magnitude of the distance between atom pairs. In fact, in a one-component system such as silicon one 


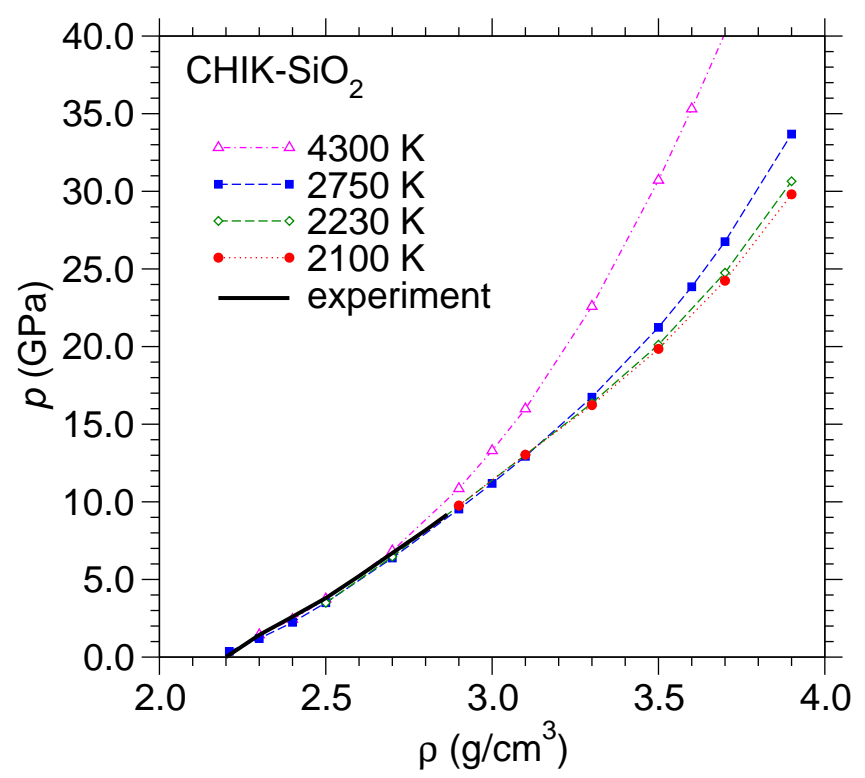

Figure 1. Pressure as a function of density for several temperatures, as indicated. The experimental data are taken from Ref. 33.

has to include at least three-body interactions to reproduce a tetrahedral network structure. However, in a binary system such as silica there is the competition between three different pair potentials. By tuning the parameters for the repulsive OO interactions and the attractive $\mathrm{SiO}$ interactions appropriately, one can generate a network structure with Si-O tetrahedra as the preferred structural units.

Apart from a rather unreliable description of the equation state, the widely used BKS potential [8 has been proven successful to describe various static and dynamic properties of amorphous silica quite reliably (see, e.g., Ref. [29, 30, 31). The parametrization of the BKS model was based on Hartree-Fock calculations of a single $\mathrm{SiO}_{4}$ tetrahedron, charge-saturated by four hydrogen atoms [8]. In contrast to that, the development of the CHIK potential was based on a CPMD simulation of a bulk system of 114 particles. A structural fitting procedure was used for the parametrization. The idea was to match the partial pair correlation functions, as obtained with the new effective potential, with those obtained from CPMD. As the BKS potential, the CHIK model consists of a long-ranged Coulomb part and a shortranged Buckingham potential,

$$
u_{\alpha \beta}(r)=\frac{q_{\alpha} q_{\beta} e^{2}}{r}+A_{\alpha \beta} \exp \left(-B_{\alpha \beta} r\right)-\frac{C_{\alpha \beta}}{r^{6}},
$$

where $r$ is the distance between an ion of type $\alpha$ and an ion of type $\beta(\alpha, \beta=\mathrm{Si}, \mathrm{O})$, $e$ is the elementary charge, and $q_{\mathrm{Si}}=1.910418$ and $q_{\mathrm{O}}=-q_{\mathrm{Si}} / 2$ are effective partial charges. The values of the parameters $\left\{A_{\alpha \beta}, B_{\alpha \beta}, C_{\alpha \beta}\right\}$ are $A_{\mathrm{SiSi}}=3150.462646 \mathrm{eV}$, $B_{\mathrm{SiSi}}=2.851451 \AA^{-1}, C_{\mathrm{SiSi}}=626.751953 \mathrm{eV} / \AA^{6}, A_{\mathrm{SiO}}=27029.419922 \mathrm{eV}$, $B_{\mathrm{SiO}}=5.158606 \AA^{-1}, C_{\mathrm{SiO}}=148.099091 \mathrm{eV} / \AA^{6}, A_{\mathrm{OO}}=659.595398 \mathrm{eV}, B_{\mathrm{OO}}=$ $2.590066 \AA^{-1}$, and $C_{\mathrm{OO}}=26.836679 \mathrm{eV} / \AA^{6}$. More details on the potential can be found in Ref. [28]. 


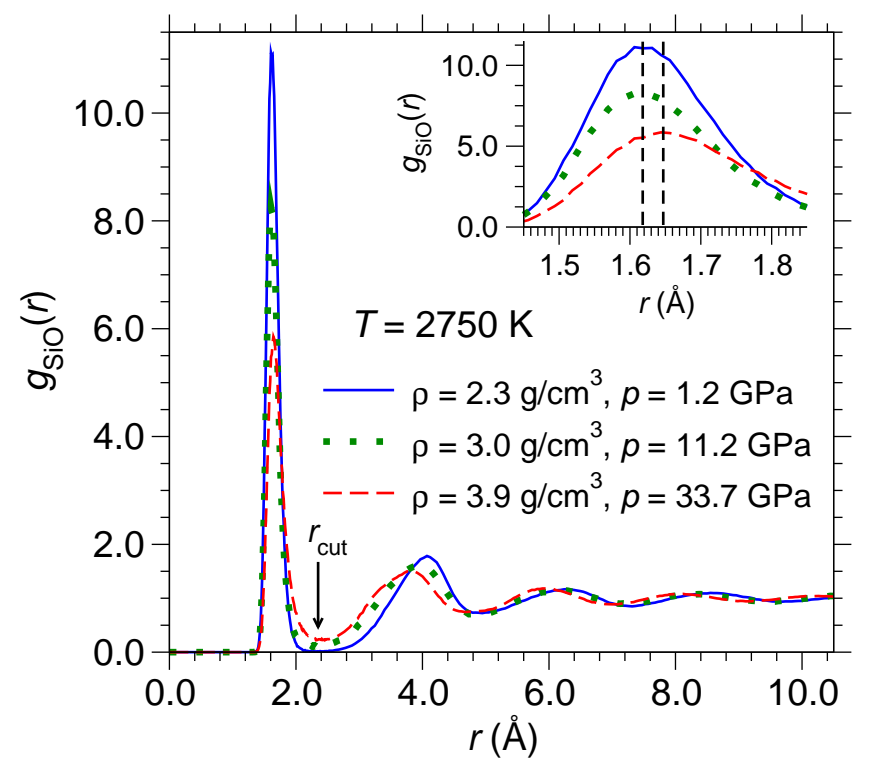

Figure 2. Partial pair correlation function for the Si-O correlations, $g_{\mathrm{SiO}}(r)$, for different densities at the temperature $T=2750 \mathrm{~K}$. The arrow indicates the cut-off $r_{\text {cut }}=2.35 \mathrm{~g} / \mathrm{cm}^{3}$, used to identify $\mathrm{SiO}$ bonds. The inset shows an enlargement of the data. The two vertical dashed lines at $1.618 \AA$ and $1.647 \AA$ indicate the location of the maxima for the densities $\rho=2.3 \mathrm{~g} / \mathrm{cm}^{3}$ and $\rho=3.9 \mathrm{~g} / \mathrm{cm}^{3}$, respectively.

MD simulations have been performed for systems of $N=1152$ particles at the densities $\rho=2.3,2.4,2.5,2.7,2.9,3.0,3.1,3.3,3.5,3.6,3.7,3.9$, and $4.2 \mathrm{~g} / \mathrm{cm}^{3}$ in the temperature range $6100 \mathrm{~K} \geq T \geq 2100 \mathrm{~K}$. The equations of motion were integrated by the velocity form of the Verlet algorithm [32] using a time step of $1.6 \mathrm{fs}$. The longrange Coulomb forces and energies were computed by the Ewald summation technique 32. At each state, the system was equilibrated in the $N V T$ ensemble during $0.2 \mathrm{~ns}$ to $25 \mathrm{~ns}$. Temperature was kept constant by coupling the system periodically to a stochastic heat bath. After the equilibration, structural and dynamic quantities were obtained from production runs in the microcanonical ensemble.

Figure 1 displays the equation of state, $p(\rho)$, along different isotherms. For pressures up to about $10 \mathrm{GPa}$, the pressure is almost independent of temperature in the range $4300 \mathrm{~K} \geq T \geq 2100 \mathrm{~K}$. In this pressure range, the simulation is in very good agreement with experiment [33]. This demonstrates the transferability of the CHIK model: this model has been parametrized from a single CPMD run at $T=3600 \mathrm{~K}$ and $\rho=2.2 \mathrm{~g} / \mathrm{cm}^{3}$, without considering states at high pressure. Thus, at least as far as the equation of state is concerned, the CHIK potential provides a reliable description and so it appears to be appropriate for the investigation of silica under high pressure.

\section{Results}

To analyze the change of the network structure of amorphous silica under compression, we consider the temperature dependence of the $\mathrm{Si}-\mathrm{O}$ as well as the O-Si coordination. 

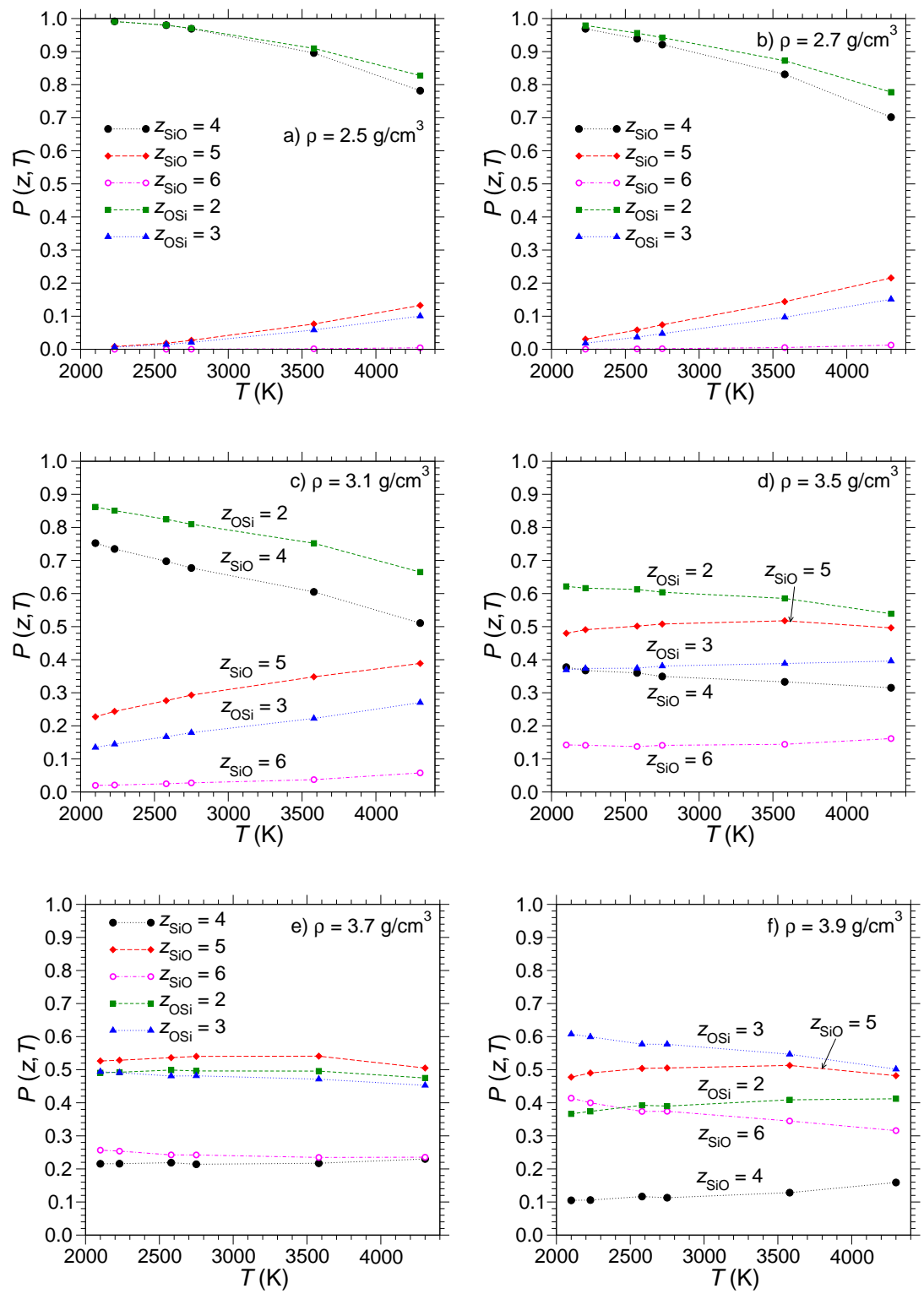

Figure 3. Temperature dependence of different $\mathrm{SiO}$ and OSi coordination numbers $z$. a) $\rho=2.5 \mathrm{~g} / \mathrm{cm}^{3}$, b) $\rho=2.7 \mathrm{~g} / \mathrm{cm}^{3}$, c) $\rho=3.1 \mathrm{~g} / \mathrm{cm}^{3}$, d) $\rho=3.5 \mathrm{~g} / \mathrm{cm}^{3}$, e) $\rho=3.7 \mathrm{~g} / \mathrm{cm}^{3}$, f) $\rho=3.9 \mathrm{~g} / \mathrm{cm}^{3}$. 


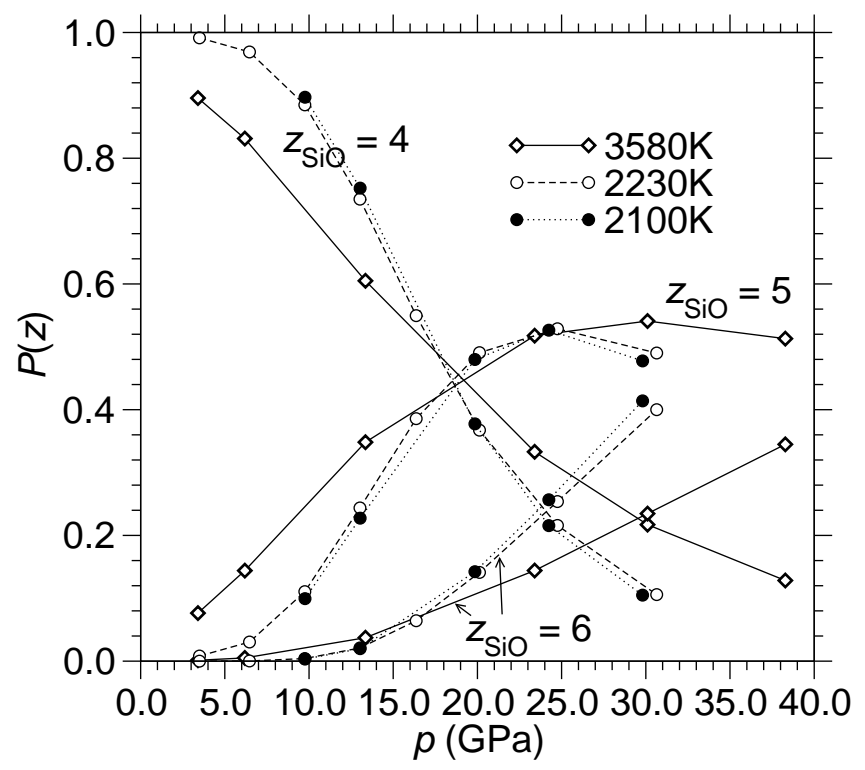

Figure 4. Pressure dependence of the Si-O coordination at $T=3580 \mathrm{~K}, 2230 \mathrm{~K}$, and $2100 \mathrm{~K}$.

To this end, coordination numbers $z_{\mathrm{SiO}}$ and $z_{\mathrm{OSi}}$ are defined as the number of $\mathrm{O}(\mathrm{Si})$ atoms surrounding a $\mathrm{Si}(\mathrm{O})$ atom within a distance $r \leq r_{\text {cut }}$. For the cut-off radius we use the value $r_{\text {cut }}=2.35 \AA$ that corresponds to the location of the first minimum of the partial pair correlation function for the Si-O correlations, $g_{\mathrm{SiO}}(r)$ [18. As Fig. [2] shows, the location of this minimum is indeed essentially independent of density. However, one can infer from the inset of Fig. 2 that the length of the $\mathrm{SiO}$ bond changes slightly from about $1.62 \AA$ at $2.3 \mathrm{~g} / \mathrm{cm}^{3}$ to about $1.65 \AA$ at $3.9 \mathrm{~g} / \mathrm{cm}^{3}$. This finding is in very good agreement with results from X-ray diffraction experiments 4. Moreover, the periodicity of the peaks in $g_{\mathrm{SiO}}(r)$ changes with density. As we shall see below, this is due to the loss of tetrahedral order at high densities, reflected, e.g., in the Fourier transform of $g_{\mathrm{SiO}}(r)$, the partial structure factor $S_{\mathrm{SiO}}(q)$, by a shift of the first sharp diffraction peak to higher wavenumbers $q$.

Figure 3 shows the probability distributions $P(z)$ at different densities for all the relevant coordination numbers $z_{\mathrm{SiO}}$ and $z_{\mathrm{OSi}}$ as a function of temperature. For the three densities $\rho=2.5,2.7$, and $3.1 \mathrm{~g} / \mathrm{cm}^{3}$, most of the Si atoms exhibit the tetrahedral four-fold coordination by $\mathrm{O}$ atoms and most of the $\mathrm{O}$ atoms serve as bridging atoms between the tetrahedra $\left(z_{\mathrm{OSi}}=2\right)$. In this case, the local defect structures that correspond to $z_{\mathrm{SiO}}=5,6$ as well as $z_{\mathrm{OSi}}=3$ become less frequent with decreasing temperature such that one expects a very low probability of these structures at low temperatures. A different behavior is seen at the density $\rho=3.5 \mathrm{~g} / \mathrm{cm}^{3}$. Now, $z_{\mathrm{SiO}}=5$ has a higher probability than the tetrahedral coordination $z_{\mathrm{SiO}}=4$ and one observes a relatively high probability of $z_{\mathrm{OSi}}=3$ and a significant number of six-fold coordinated $\mathrm{Si}$ atoms. One can hardly extrapolate the curves for $3.5 \mathrm{~g} / \mathrm{cm}^{3}$ to lower temperatures since at this density all the coordination numbers show a rather weak temperature dependence in the considered temperature range. The same trend is also seen at the 

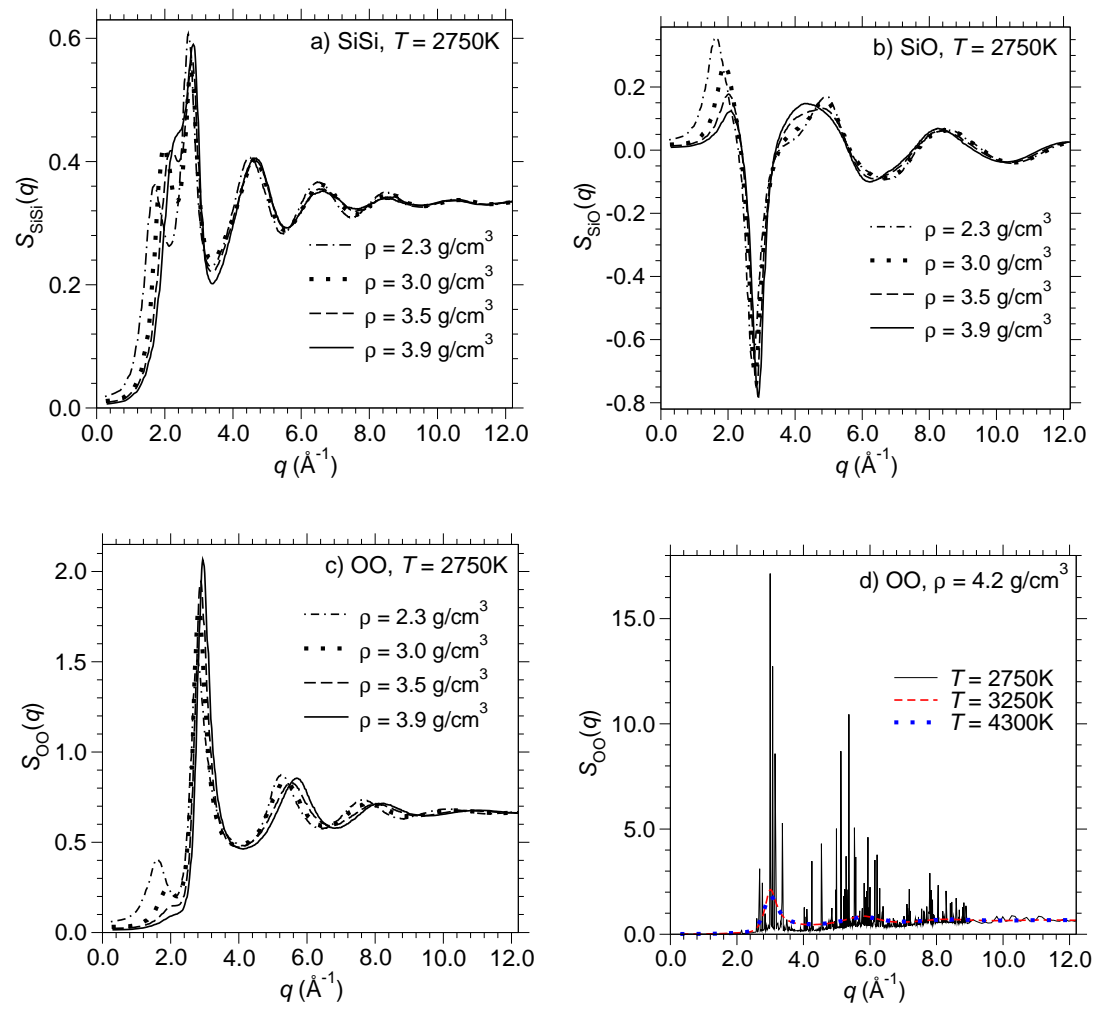

Figure 5. Partial static structure factors $S_{\alpha \beta}(q)(\alpha, \beta=\mathrm{Si}, \mathrm{O})$ for different densities at the temperature $T=2750 \mathrm{~K}$. a) $S_{\mathrm{SiSi}}(q)$, b) $S_{\mathrm{SiO}}(q)$, c) $S_{\mathrm{OO}}(q)$. In panel d), $S_{\mathrm{OO}}(q)$ for $\rho=4.2 \mathrm{~g} / \mathrm{cm}^{3}$ at three different temperatures is shown, indicating that the system has crystallized at $T=2750 \mathrm{~K}$.

two high densities, $\rho=3.7 \mathrm{~g} / \mathrm{cm}^{3}$ and $\rho=3.9 \mathrm{~g} / \mathrm{cm}^{3}$. Whereas the number of five-fold coordinated $\mathrm{Si}$ atoms keeps approximately constant around 50\%, the coordination number $z_{\mathrm{SiO}}=6$ further increases, whereas the tetrahedral coordination $z_{\mathrm{SiO}}=4$ becomes less and less important with increasing density. At $\rho=3.9 \mathrm{~g} / \mathrm{cm}^{3}$, there are more oxygen atoms that are three-fold coordinated by Si atoms than bridging oxygens.

The $\mathrm{SiO}$ coordination as a function of pressure is shown in Fig. 4 for $T=2100 \mathrm{~K}$, $2230 \mathrm{~K}$ and $3580 \mathrm{~K}$. Although the qualitative behaviour of $P\left(z_{\mathrm{SiO}}\right)$ is similar at the different temperatures, there are significant quantitative differences between the coordination numbers at $T=3580 \mathrm{~K}$ and those at the two lower temperatures. The maximum of $P\left(z_{\mathrm{SiO}}=5\right)$ shifts from about $30 \mathrm{GPa}$ at $3580 \mathrm{~K}$ to about $25 \mathrm{GPa}$ at the two lower temperatures. Moreover, at low temperatures, $P\left(z_{\mathrm{SiO}}=4\right)$ decays more rapidly, accompanied by a faster increase of $P\left(z_{\mathrm{SiO}}=6\right)$. Note that in recent simulation studies using both ab initio techniques and classical MD simulation 25, 26, the pressure dependence of the $\mathrm{SiO}$ coordination has been only discussed for temperatures around $3500 \mathrm{~K}$. However, Fig. 4 demonstrates that at least in the temperature range between 2000 to $4000 \mathrm{~K}$ the temperature dependence of $P\left(z_{\mathrm{SiO}}\right)$ has to be taken into account to allow for a quantitative comparison with experimental data, done at much lower temperatures. 

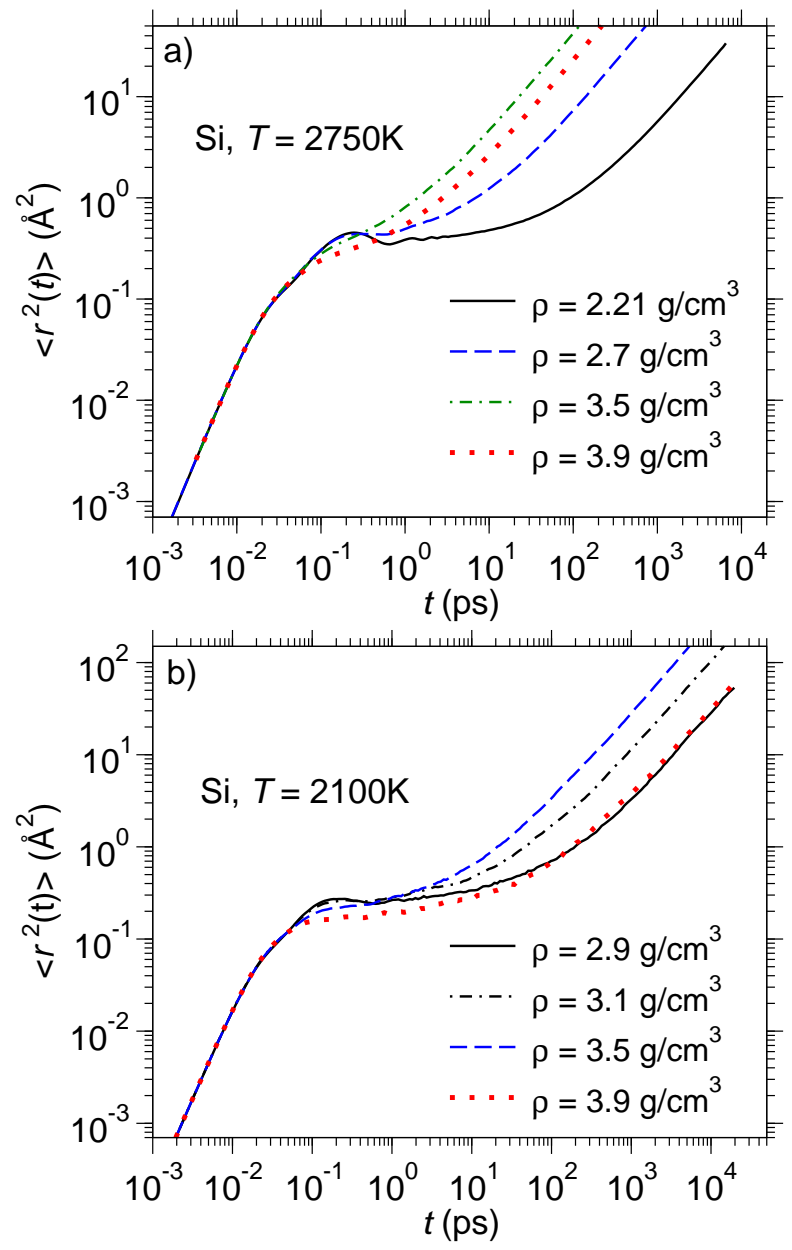

Figure 6. Mean squared displacements for the silicon atoms for different densities at a) $T=2750 \mathrm{~K}$ and b) $T=2100 \mathrm{~K}$.

The inspection of the $\mathrm{SiO}$ and $\mathrm{OSi}$ coordination number distributions indicates that significant changes occur in the network structure for pressures above, say $10 \mathrm{GPa}$. This finding is similar to what has been observed experimentally for silica glasses. At pressures above about $20 \mathrm{GPa}$ a network with five- and six-fold coordinated Si atoms is formed, similar to what has been recently found in other simulation studies [10, 25, 26. It remains an open question whether in silica a first-order phase transition from a low density liquid to a high density liquid exists. Our data in Figs. 3 and 4 is also consistent with a gradual crossover from a low density phase (with $z_{\mathrm{SiO}}=4$ ) to a high density phase (with $z_{\mathrm{SiO}}=6$ ), occurring at low temperatures.

To investigate the changes in the network structure on intermediate length scales, we now consider the partial static structure factors, defined as [18]

$$
S_{\alpha \beta}(q)=\frac{1}{N} \sum_{k=1}^{N_{\alpha}} \sum_{l=1}^{N_{\beta}}\left\langle\exp \left(i \mathbf{q} \cdot \mathbf{r}_{k l}\right)\right\rangle \quad \alpha, \beta=\mathrm{Si}, \mathrm{O},
$$


with $N_{\alpha}$ the number of atoms of type $\alpha$, $\mathbf{q}$ the wavevector, and $\mathbf{r}_{k l}=\mathbf{r}_{k}-\mathbf{r}_{l}$ the distance vector between particle $k$ and particle $l$.

Fig. 5 shows the three $S_{\alpha \beta}(q)$ at the temperature $T=2750 \mathrm{~K}$ for different densities. For the density $2.3 \mathrm{~g} / \mathrm{cm}^{3}$ the first peak [also called first sharp diffraction peak (FSDP)] is located at a wavenumber of about $1.6-1.7 \AA^{-1}$. This peak is due to the tetrahedral order present in "low-density" silica. Its location is related to the length scale of two connected tetrahedra [30. With increasing density, the first peak shifts to higher values of $q$ and its amplitude decreases significantly. The behavior of the FSDP reflects the change of the structure from an open tetrahedral network at low densities to a more densely packed network at high densities, provided by $\mathrm{SiO}_{5}$ and $\mathrm{SiO}_{6}$ units as well as oxygen atoms that are three-fold coordinated by silicon atoms. Note that the partial structure factors $S_{\alpha \beta}(q)$ are the required input for calculations in the framework of the mode-coupling theory of the glass transition [34, MCT, from which the dynamics of the melt can be predicted. A MCT calculation based on the $S_{\alpha \beta}(q)$ of our simulation is presented elsewhere in this issue [35].

Figure $5 \mathrm{H}$ shows $S_{\mathrm{OO}}(q)$ for the density $\rho=4.2 \mathrm{~g} / \mathrm{cm}^{3}$ at three different temperatures. At this high density, the system crystallizes at a temperature of $T=2750 \mathrm{~K}$ which is reflected by the occurrence of Bragg peaks in $S_{\mathrm{OO}}(q)$. At a temperature of $T=3250 \mathrm{~K}$ the system remains liquid; the structure factor in this case reminds one to that of a simple liquid, showing no sign of the FSDP feature.

The structural changes in the network with increasing pressure are accompanied by an anomalous behavior of relaxation processes. This can be inferred from the mean squared displacements of a tagged particle of type $\alpha=\mathrm{Si}, \mathrm{O},\left\langle r_{\alpha}^{2}(t)\right\rangle=$ $\left(1 / N_{\alpha}\right) \sum_{k=1}^{N_{\alpha}}\left\langle\left(\mathbf{r}_{k}(t)-\mathbf{r}_{k}(0)\right)^{2}\right\rangle$. In Fig. [6 only $\left\langle r_{\mathrm{Si}}^{2}(t)\right\rangle$ is shown, since the mean squared displacement for oxygen exhibits a similar behaviour. At $T=2750 \mathrm{~K}$, several time regimes can be clearly distinguished at the low density $\rho=2.21 \mathrm{~g} / \mathrm{cm}^{3}$, consisting of a ballistic regime at very short times $\left(\left\langle r^{2}(t)\right\rangle \propto t^{2}\right)$, an intermediate regime with a maximum $0.2 \mathrm{ps}$, a plateau-like region and finally a linear regime, $\left\langle r^{2}(t)\right\rangle \propto t^{2}$, indicating diffusive behaviour. Note that the maximum at $0.2 \mathrm{ps}$ is both related to the so-called boson peak and to finite size effects present in the dynamics of silica (for details see Refs. [12, 31). That this feature is not seen at high density might be related to the changes occurring in the network structure. From $\rho=2.21 \mathrm{~g} / \mathrm{cm}^{3}$ to $\rho=3.5 \mathrm{~g} / \mathrm{cm}^{3}$, an acceleration of the diffusion dynamics can be inferred from Fig. 6 $\mathrm{a}$, followed by the opposite behaviour for a further decrease of the density. The same behaviour as for $T=2750 \mathrm{~K}$ is also seen for the lower temperature, $T=2100 \mathrm{~K}$, although the effect of a maximal speed of diffusion is now more pronounced (Fig. 6b). One can also infer from Fig. 6b that the particles become more localized with increasing density, indicated by a decrease of the plateau's height with increasing density.

The self-diffusion constants can be easily determined from the mean squared displacements using the Einstein relation, $D_{\alpha}=\lim _{t \rightarrow \infty}\left\langle r_{\alpha}^{2}(t)\right\rangle /(6 t)$. In Fig. [7, the self-diffusion constant for silicon, $D_{\mathrm{Si}}$, along different isotherms is displayed as a function of density and pressure. Note that $D_{\mathrm{O}}$, albeit slightly larger, exhibits the same behaviour. Along the different isotherms a maximum is found around about $3.5 \mathrm{~g} / \mathrm{cm}^{3}$, corresponding to a pressure of about $20 \mathrm{GPa}$ (see Fig. $7 \mathrm{~b}$ ). Thereby, the diffusivity maximum becomes more pronounced with decreasing temperature. The behaviour of $D_{\mathrm{Si}}$ is closely related to that of the coordination numbers. Above about $p=20 \mathrm{GPa}$, the location of the diffusivity maximum, the number of five-fold coordinated $\mathrm{Si}$ atoms roughly shows a saturation, while the number of six-fold coordinated Si atoms further increases with increasing pressure. It seems that a $\mathrm{SiO}$ network with many five-fold 

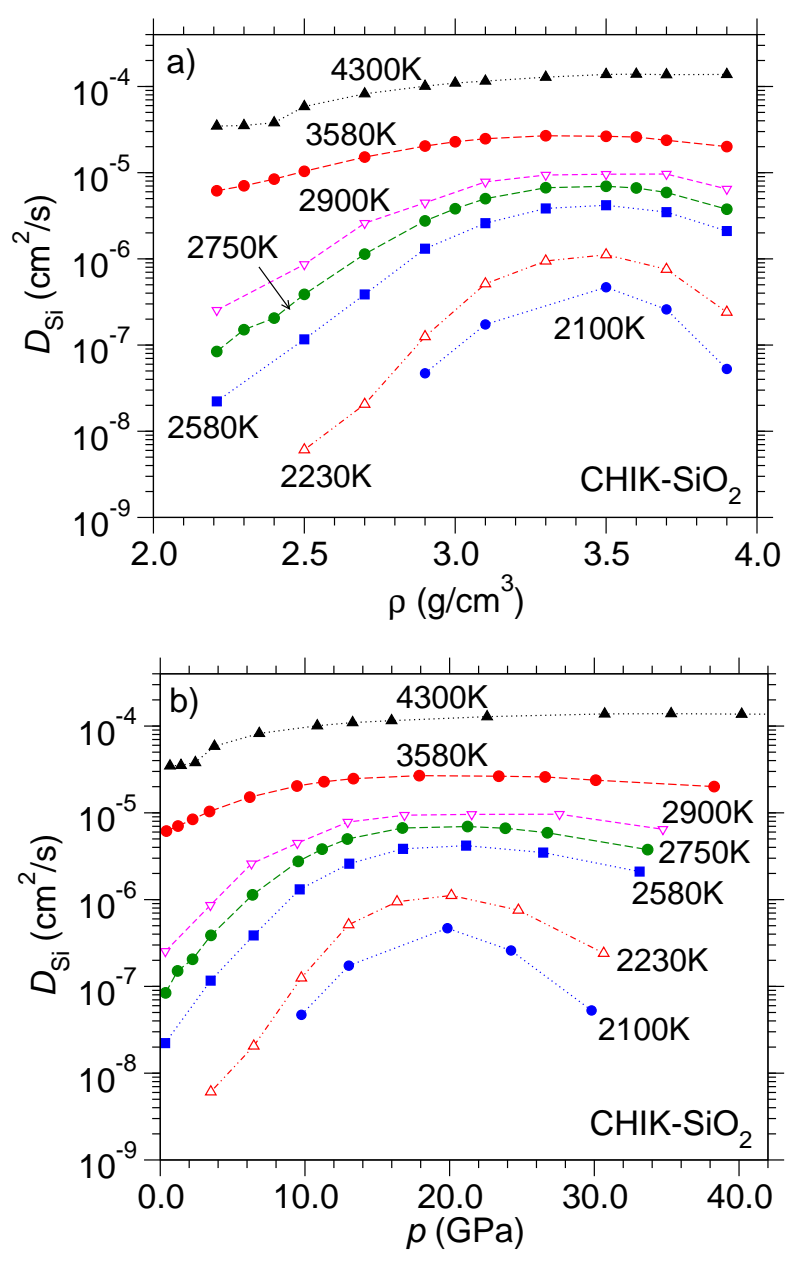

Figure 7. Self-diffusion constant of silicon as a function of density along different isotherms as a function of density, a), and as a function of pressure, b).

coordinated $\mathrm{Si}$ atoms tends to have a faster diffusion dynamics than one with many sixfold coordinated $\mathrm{Si}$ atoms. Therefore, for pressures up to about $20 \mathrm{GPa}$ the network dynamics is dominated by an increasing number of five-fold coordinated $\mathrm{Si}$ atoms, leading to an acceleration of diffusion. However, at pressures above about $20 \mathrm{GPa}$, the network dynamics is dominated by an increasing number of $\mathrm{SiO}_{6}$ units, leading to a decrease of the diffusion constants with pressure. Thus, the diffusivity maximum is due to an interplay between five- and six-fold coordinated Si atoms.

\section{Conclusions}

Results of an extensive MD simulation have been presented to study structural and dynamic properties of liquid silica under (high) pressure. As an interaction potential a simple pair potential, the so-called CHIK potential, was used that leads to a reliable description of the equation of state. We have shown that the change of the network 
structure with increasing pressure is related to an anomalous diffusion dynamics, characterized by a diffusivity maximum around $20 \mathrm{GPa}$. Elsewhere in this issue [35], a calculation in the framework of mode coupling theory based on the structural input of our simulation is presented that explicitly shows that the changes in the network structure are intimately related to the anomalous transport in amorphous silica. It is still an open question whether, at low temperatures, there is a first-order phase transition from a low-density to a high-density transition in silica. This question will be addressed in future studies with the CHIK potential.

\section{Acknowledgments}

We thank Schott Glas for financial support. Computing time on the JUMP at the NIC Jülich is gratefully acknowledged.

\section{References}

[1] Grimsditch M 1984 Phys. Rev. Lett. 522379

[2] Hemley R J, Mao H K, Bell P M and Mysen B O 1986 Phys. Rev. Lett. 57747

[3] Williams Q and Jeanloz R 1988 Science 239902

[4] Meade C, Hemley R J and Mao H K 1992 Phys. Rev. Lett. 691387

[5] Zha C, Hemley R J, Mao H, Duffy T S and Meade C 1994 Phys. Rev. B 5013105

[6] Brazhkin V V and Lyapin A G 2003 J. Phys.: Condens. Matter 156059

[7] Lin J-F, Fukui H, Prendergast D, Okuchi T, Cai Y Q, Hiraoka N, Yoo C-S, Trave A, Eng P, Hu M Y and Chow P, 2007 Phys. Rev. B 75012201

[8] van Beest B W H, Kramer G J and van Santen R A 1990 Phys. Rev. Lett. 641955

[9] Tse J S, Klug D D and Le Page Y 1992 Phys. Rev. B 465933

[10] Barrat J-L, Badro J and Gillet Ph 1997 J. Comp. Simul. 2017

[11] Hemley R J, Badro J and Teter D M 2000 in Physics Meets Mineralogy - Condensed Matter Physics in Geosciences ed Aoki H, Syono Y and Hemley R J (Cambridge: Cambridge University Press) p 173

[12] Horbach J, Kob W, Binder K and Angell C A 1996 Phys. Rev. E 54 R5897

[13] Kushiro I 1978 Earth Planet. Sc. Lett. 4187

[14] Rubie D C, Ross C R, Carroll M R and Elphick S C 1993 Am. Mineral. 78574

[15] Poe B T, McMillan P F, Rubie D C, Chakraborty S, Yarger J and Diefenbacher J 1997 Science 2761245

[16] Tinker D, Lesher C E and Hutcheon I D 2003 Geochim. Cosmochim. Acta 67133

[17] Angell C A 1985 in Relaxation in Complex Systems ed Ngai K and Wright G B (Springfield: US Dept. of Commerce)

[18] Binder K and Kob W 2005 Glassy Materials and Disordered Solids: An Introduction to Their Statistical Mechanics (Singapure: World Scientific)

[19] Angell C A, Cheeseman P A and Tammadon S 1992 Science 218885

[20] Saika-Voivod I, Sciortino F and Poole P H 2000 Phys. Rev. E 63011202

[21] Scott Shell M, Debenedetti P G and Panagiotopoulos A Z 2002 Phys. Rev. E 66011202

[22] Sharma R, Mudi A and Chakravarty C, 2006 J. Chem. Phys. 125044705

[23] Le Grand A, Dreyfus C, Bousquet C and Pick R M, 2007 Phys. Rev. E 75061203

[24] Kohn W 1996 in Monte Carlo and Molecular Dynamics of Condensed Matter Systems ed Binder K and Ciccotti (Bologna: Società Italiana di Fisica) p 561; Car R ibid. p 601

[25] Trave A, Tangney P, Scandolo S, Pasquarello A and Car R 2002 Phys. Rev. Lett. 89245504

[26] Tangney P and Scandolo S 2002 J. Chem. Phys. 1178898

[27] Herzbach D, Binder K and Müser M H 2005 J. Chem. Phys. 123124711

[28] Carré A, Horbach J, Ispas S and Kob W 2008 Europhys. Lett. 8217001

[29] Vollmayr K, Kob W and Binder K 1996 Phys. Rev. B 5415808

[30] Horbach J and Kob W 1999 Phys. Rev. B 603169

[31] Horbach J, Kob W and Binder K 2001 Eur. Phys. J. B 19531

[32] Allen M and Tildesley D 1987 Compute Simulation of Liquids (Oxford: Oxford University Press)

[33] Tsiok O B, Brazhkin V V, Lyapin A G and Khvostantsev L G 1998 Phys. Rev. Lett. 80999

[34] Götze W 1999 J. Phys.: Condens. Matter 11 A1 
[35] Voigtmann Th and Horbach J 2008 J. Phys.: Condens. Matter this issue 